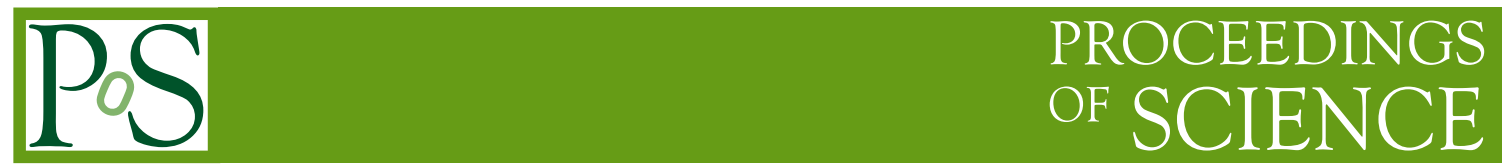

\title{
Top production and decay
}

\section{Sven Menke ${ }^{a, *}$ on behalf of the ATLAS, CMS and LHCb collaborations}

${ }^{a}$ Max-Planck-Institut für Physik,

Föhringer Ring 6, 80805 München, Germany

E-mail: menke@mppmu.mpg.de

Measurements of inclusive and differential production cross-sections of top-quarks in pairs and in single top-quark modes as well as properties of their subsequent decays at the LHC are presented. Recent analyses of proton-proton collisions recorded by the ATLAS, CMS and LHCb detectors at centre-of-mass energies of 5, 7,8 and $13 \mathrm{TeV}$ and combinations of the results are discussed. Among the properties analysed are tests of lepton universality, the W-boson helicity, tests of $C \mathcal{P}$ violation, studies of b-quark-fragmentation, top-quark polarisation measurements and the mass of the top-quark.

The Ninth Annual Conference on Large Hadron Collider Physics - LHCP2021

7-12 June 2021

Online

${ }^{*}$ Speaker 


\section{Introduction}

Top quarks are copiously produced in proton-proton collisions at the Large Hadron Collider (LHC) at centre-of-mass energies ranging from 5 to $13 \mathrm{TeV}$ and recorded by the ATLAS [1], CMS [2] and LHCb [3] experiments in the years 2011-2018. Inclusive and differential crosssection measurements of top-quark production in pairs and in single-top events as discussed in section 2 allow precision comparisons with predictions at next-to-leading (NLO) and next-to-nextto-leading order (NNLO) accuracy in Quantum Chromo Dynamics (QCD). The decay of top-quarks offers a rich laboratory for a variety of properties discussed in section 3 including the mass of this most massive of known fundamental particles - now reaching $0.5 \mathrm{GeV}$ precision.

\section{Production of top quarks}

Inclusive production cross-section measurements in proton-proton collisions at the LHC involving top-quarks are now spanning over 5 orders of magnitude - with top-quark pair production on the high end and the recently established evidence for the production of 4 top-quarks [4] on the low end.

\subsection{Pair production cross-sections of top quarks}

The most recent analyses of inclusive top-quark pair-production cross-sections have been made by ATLAS [5] and CMS [6] on very small $\left(257 \mathrm{pb}^{-1}\right.$ and $304 \mathrm{pb}^{-1}$, respectively) datasets taken in 2017 at $\sqrt{s}=5.02 \mathrm{TeV}$. Compared to the much larger $\sqrt{s}=13 \mathrm{TeV}$ datasets, the lower centre-ofmass point offers a very low amount of additional soft collisions per event and an about 2.3 times larger fraction of quark-antiquark-annihilation (instead of gluon initiated) processes. The di-lepton channel has been used by ATLAS and the $\mathrm{e}^{ \pm} \mu^{\mp}$ di-lepton subset by CMS. After correcting to the full phase-space and all decay channels the results obtained are:

$\sigma_{\mathrm{tt}}(5.02 \mathrm{TeV})^{\mathrm{ATLAS}}=66.0 \pm 4.5_{\text {stat }} \pm 1.6_{\text {syst }} \pm 1.2_{\text {lumi }} \pm 0.2_{\text {beam }} \mathrm{pb}=66.0 \pm 4.9_{\text {tot }} \mathrm{pb}$ $\sigma_{\text {tit }}(5.02 \mathrm{TeV})^{\mathrm{CMS}}=60.3 \pm 5.0_{\text {stat }} \pm 2.8_{\text {syst }} \pm 0.9_{\text {lumi }} \mathrm{pb}=60.3 \pm 5.5_{\text {tot }} \mathrm{pb}$.

Both results agree well with each other and with the theoretical predictions at NNLO and nextto-next-to-leading-log (NNLL) accuracy in QCD. The uncertainties are dominated by the small statistics (stat) while luminosity (lumi) and beam-energy (beam) related uncertainties are explicitly given and small. Among the largest contributions to the remaining systematic uncertainties (syst) is the modelling of the tit-events due to the restriction to the di-leptonic final state and the impact on the transverse momentum of the selected leptons. Figure 1 shows all inclusive pair-production results from the LHCTopWG [7] and the combined Tevatron proton-antiproton data as function of $\sqrt{s}$ compared to NNLO+NNLL predictions [8]. LHCb measured forward top-quark-pair production in the fiducial phase-space $(\simeq 2.0<\eta<4.5)$ at $\sqrt{s}=8 \mathrm{TeV}$ [9] and $13 \mathrm{TeV}$ [10] also in agreement with Standard Model (SM) expectations - but due to the small fiducial forward phase-space these are about $\simeq 0.02 \%$ of the fully inclusive cross-sections in magnitude at the respective centre-of-mass energies. Single- and double-differential cross sections for top-quark pair production are analysed by ATLAS [11] and CMS [12] and recent results at $\sqrt{s}=13 \mathrm{TeV}$ for the full phase-space unfolded to either parton-level or particle-level are compared to NLO simulations - including parton-showering for the comparison on particle-level. 


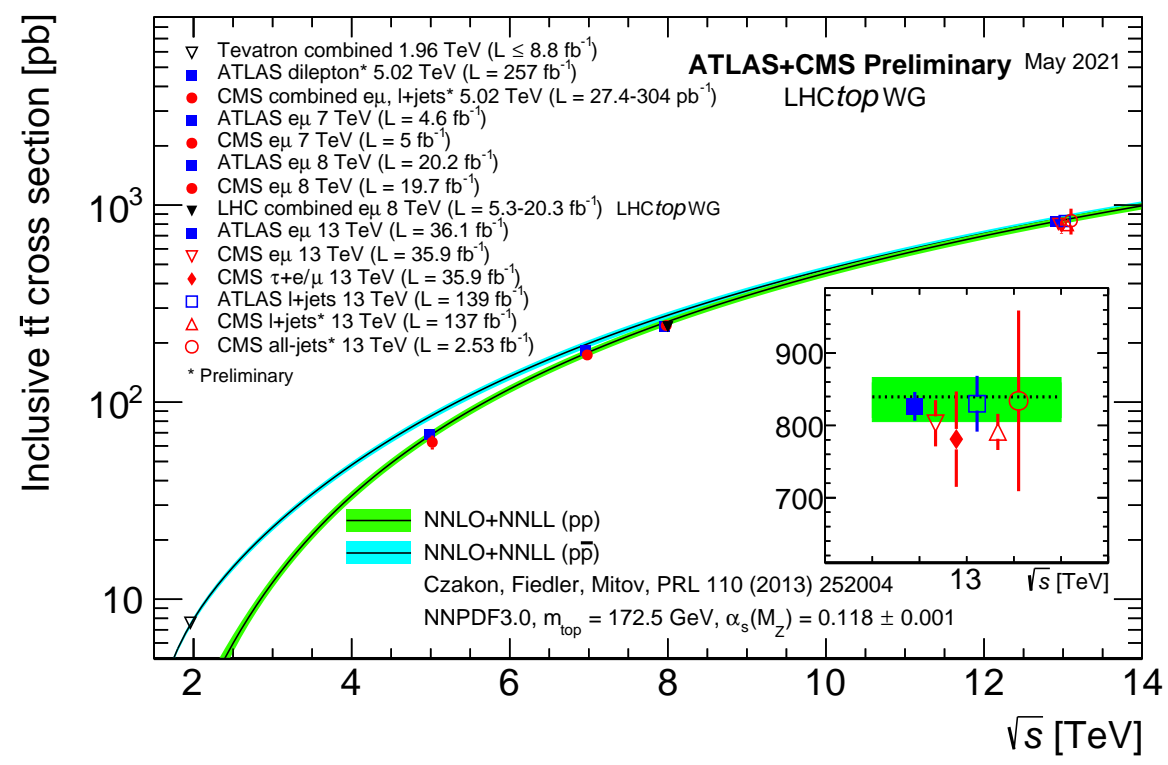

Figure 1: Inclusive top-quark pair-production cross-sections in pp and $p \bar{p}$ collisions as function of $\sqrt{s}$ from ATLAS, CMS at the LHC and the Tevatron experiments D0 and CDF as compiled by the LHCTopWG [7]. The data are compared to NNLLO+NNLL QCD calculations [8].

CMS categorises events in the lepton+jets channel by the number of resolved or boosted topquark candidates and the number of b-quark-tags while ATLAS uses all-hadronic events in the fully resolved mode with a data-driven background estimation method in 5 regions besides the signal region based on the number of b-quark-tags and the top-quark-candidate mass. Distributions are unfolded to particle- and parton-level and compared to NLO simulations: POWHEG [13] interfaced to PYTHIA [14] (this is the default choice) or HERWIG [15] for parton-showering and MG5 aMC@NLO [16] + PYTHIA. ATLAS also compares to SHERPA [17]. Unfolding to particle-level is done with detector simulations using GEANT4 [18, 19] while unfolding to parton-level is done with PYTHIA [14]. All NLO simulations tend to predict too hard transverse momenta for individual top-quarks and the $\mathrm{t} \mathrm{t}$-system and also deviate at large absolute values of top-quark rapidity. The mass distribution of the $\bar{t} \bar{t}$-system is wider in data than predicted by the NLO simulations. For ATLAS, the overall agreement is best for POWHEG + HERWIG and MG5aMC@NLO + PYTHIA is most discrepant. Single differential distributions are better modelled than the double-differential ones for all NLO generators. On parton-level, CMS also compares to MATRIX [20] calculations at NNLO QCD accuracy and sees in general better agreement in the phase-space regions where the NLO predictions deviate - a clear sign that higher order calculations are needed to improve the modelling of top-quark production as shown in figure 2 for the transverse momentum of the hadronically decaying top-quark. ATLAS unfolds also in the angular distance $(\Delta R)$ of the highest transverse momentum jet not part of the $\mathrm{tt}-$-system w.r.t. the leading jet in transverse momentum in the event. This distribution (see figure 3 ) has a narrow peak at $\Delta R=0$, where the extra-jet itself is the leading one and a broad peak around $\Delta R \simeq \pi$ if the leading jet stems from the tit-system. The angular distance of this second group is underestimated by most predictions. 


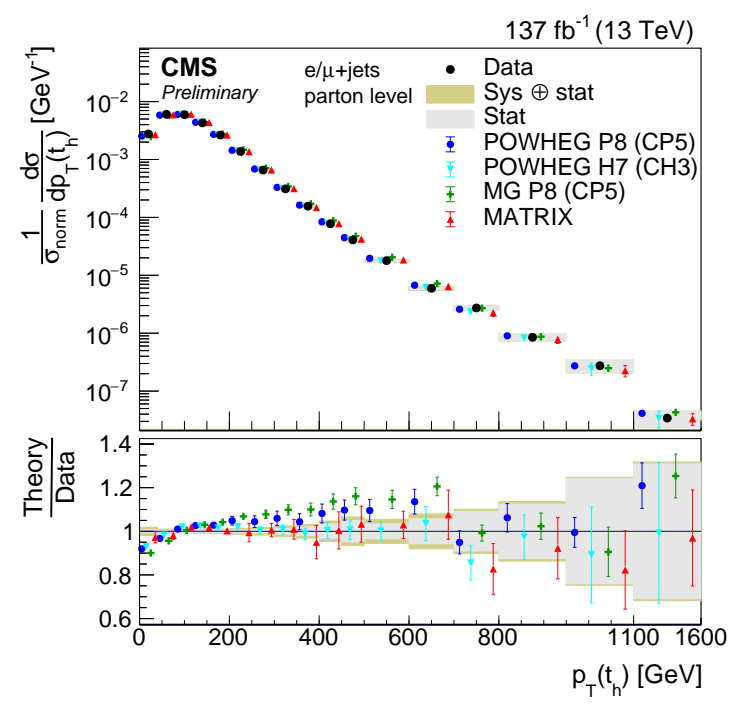

Figure 2: Transverse momentum of the hadronically decaying top-quark in $\mathrm{t} \overline{\mathrm{t}}$-events at $\sqrt{s}=13 \mathrm{TeV}$ from CMS [12] unfolded to parton-level.

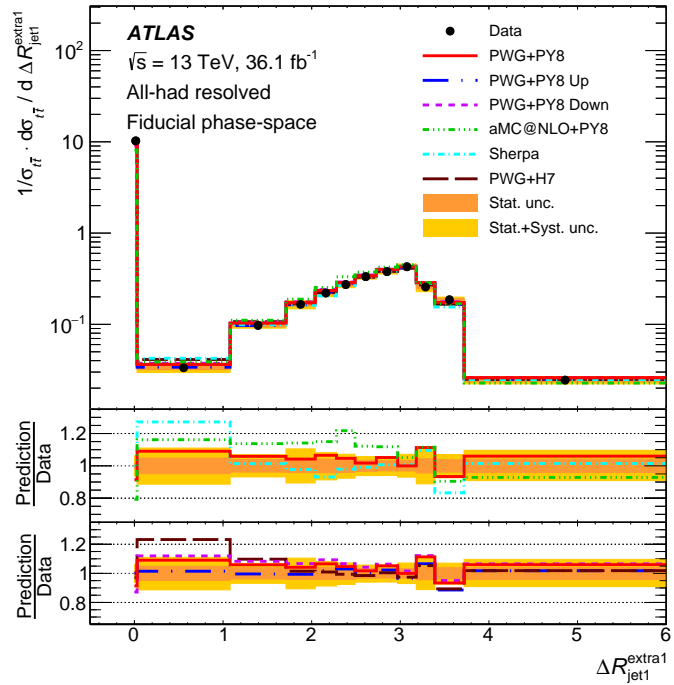

Figure 3: Angular distance of the highest transverse momentum jet not part of the $\bar{t} \bar{t}$-system to the leading jet in $\mathrm{t} \bar{t}$-events at $\sqrt{s}=13 \mathrm{TeV}$ from ATLAS [11] unfolded to particle-level.

\subsection{Single top-quark production cross-sections}

Single top-quark associated production is dominated by the $t$-channel exchange of a W-boson between quarks from the first family and a b-quark. The $s$-channel production cross-section via the $\mathrm{W}$-boson is $\simeq 20 \times$ smaller compared to $t$-channel production, while the cross-section of associated production of a t-quark with a W-boson is only $\simeq 3 \times$ smaller than $t$-channel production. However, the presence of the additional W-boson in the final state makes this channel particularly difficult to separate from top-quark pair events which are produced an order of magnitude more frequently at $\sqrt{s}=13 \mathrm{TeV}$. Recent analyses of the associated production of a single top-quark with a W-boson include the ATLAS measurement [21] at $\sqrt{s}=8 \mathrm{TeV}$ and the CMS measurement [22] at $\sqrt{s}=13 \mathrm{TeV}$ - both in the single-lepton + jets channel. Even after selection cuts on the lepton, the missing transverse momentum and after requiring exactly one b-quark-tag among the 3 jets, the signal regions remain dominated by $\mathrm{t} \overline{\mathrm{t}}$-events. Control regions with 2 b-quark-tags (ATLAS) or 4 jets (CMS) are even more $\bar{t}-$-enhanced and serve to constrain this main background. CMS uses in addition a 2 jet control region for the $\mathrm{W}+$ jet and multi-jet background. The latter being estimated in a data-driven method by inverting the lepton isolation requirement. A neural network (NN) is used by ATLAS to discriminate between signal and background based on a set of kinematic variables excluding the mass of the hadronically decaying $\mathrm{W}$-boson which is then used together with the NN-output in a final two-dimensional likelihood fit to determine the cross-section: $\sigma_{\mathrm{Wt}}(8 \mathrm{TeV})^{\mathrm{ATLAS}}=26 \pm 7_{\text {tot }} \mathrm{pb}$. CMS uses a boosted decision tree (BDT) based on kinematic variables including the mass of the hadronically decaying W-boson for signal/background discrimination and performs a binned likelihood fit on the BDT-output to extract $\sigma_{\mathrm{Wt}}(13 \mathrm{TeV})^{\mathrm{CMS}}=89 \pm 4_{\text {stat }} \pm 12_{\text {syst }}$ pb. Both are dominated by systematic uncertainties stemming from the background estimation, the jet energy 
scale, b-quark-tagging (ATLAS) or tit-modelling (CMS). Like for the pair-production of t-quarks, the agreement of inclusive single-top-quark production cross-sections with predictions at NLO+NNLL in $\mathrm{QCD}\left(\sigma_{\mathrm{Wt}}(8 \mathrm{TeV})^{\mathrm{th}}=22.4 \pm 1.5 \mathrm{pb}[23,24]\right.$ and $\left.\sigma_{\mathrm{Wt}}(13 \mathrm{TeV})^{\mathrm{th}}=71.7 \pm 3.8 \mathrm{pb}[25]\right)$ is good.

\section{Properties in top-quark decays}

Being the heaviest of all quarks and even of all known fundamental particles, the top-quarkmass is one of the most important parameters of the SM. However, the large datasets collected by the LHC experiments allows for many more measurements of top-quark-decay properties. A few recent analyses of the top-quark-mass and other properties are covered in the following sections.

\subsection{Lepton universality}

The di-lepton sample of tî-events at $\sqrt{s}=13 \mathrm{TeV}$ has been used by ATLAS [26] with a "tag and probe" approach to measure $R(\tau / \mu)$, the ratio of branching ratios of W-bosons to $\tau$ and $\mu$, respectively - thus testing the universality of weak lepton couplings to the $2 \mathrm{nd}$ and $3 \mathrm{rd}$ family for the first time at the LHC. One lepton (either $\mu$ or e) serves as "tag" and another $\mu$ as "probe" to count in an un-biased manner the number of prompt $\mathrm{W} \rightarrow \mu v_{\mu}$ and non-prompt $\mathrm{W} \rightarrow \tau v_{\tau} \rightarrow \mu v_{\mu} v_{\tau}$ decays. In addition to the two isolated leptons, the event selection requires two b-quark-tagged jets and a Z-boson mass veto for $\mu \mu$-events. $\mathrm{Z} \rightarrow \mu \mu$-events form a calibration sample by reversing the Z-mass veto and without applying any jet requirement. A special transverse impact parameter $\left|d_{0}^{\mu}\right|$, defined w.r.t. the beam-line instead of a primary vertex, is used to distinguish prompt from nonprompt muons. Finally, a likelihood fit to templates in $\left|d_{0}^{\mu}\right|$ for prompt (from the $\mathrm{Z} \rightarrow \mu \mu$ sample) and non-prompt $\left(\tau \rightarrow \mu v_{\mu} v_{\tau}\right)$ and fakes is performed to give: $R(\tau / \mu)=0.992 \pm 0.007_{\text {stat }} \pm 0.011_{\text {syst }}$, where the first error is statistical and the second from systematic uncertainties (from the prompt $\left|d_{0}^{\mu}\right|$-templates and event modelling parameters). The ratio is consistent with the SM expectation of unity and reduces the uncertainty compared to previous measurements at LEP [27] by more than a factor of 2 .

\subsection{W-boson helicity}

The SM expectation of $V-A$ structure in the $\mathrm{t} \rightarrow \mathrm{Wb}$ decay was one of the first top-quark decay properties tested at the Tevatron and later at the LHC. Most recently a combination [28] of ATLAS and CMS measurements with careful evaluation of correlations aimed to improve the experimental precision $(\simeq 3-5 \%$ for each measurement) compared to the theoretical uncertainty $(\simeq 2 \%)$. The decay is typically described by the fraction of W-bosons with longitudinal $\left(F_{0}\right)$, left-handed $\left(F_{\mathrm{L}}\right)$ and right-handed $\left(F_{\mathrm{R}}\right)$ polarisation, which add to unity and where $F_{\mathrm{R}}^{\mathrm{SM}}=0$ vanishes in the SM. This leads to a high anti-correlation of $F_{0}$ and $F_{\mathrm{L}}$ within one measurement, while the correlations between different measurements in tit-events are in the 30-40\% range. The single-top measurement by CMS is found to be $20 \%$ correlated to CMS' $\mathrm{t} \overline{\mathrm{t}}$-results - and even smaller to the tît-measurement from ATLAS. Combined fractions are: $F_{0}=0.693 \pm 0.009_{\text {stat }+ \text { bkg }} \pm 0.011_{\text {syst }}\left( \pm 2.0 \% \%_{\text {tot }}\right), F_{\mathrm{L}}=$ $0.315 \pm 0.006_{\text {stat }+\mathrm{bkg}} \pm 0.009_{\text {syst }}( \pm 3.5 \%$ tot $)$, with a total correlation of $\rho=-85 \%$. From the unitarity constraint the right-handed fraction is then: $F_{\mathrm{R}}=-0.008 \pm 0.005_{\text {stat }+ \text { bkg }} \pm 0.006_{\text {syst }}$. Statistical uncertainties cover data and background estimates while the systematic errors are dominated by radiation and scales modelling and the size and choice of simulation samples. Limits on beyond SM 
(BSM) anomalous couplings - such as right-handed vector couplings and left- and right-handed tensor couplings are improved and compatible with the SM expectation of 0.

\subsection{Tests of $C \mathcal{P}$-violation}

Levi-Civita tensors $O_{1}=\varepsilon\left(p(\mathrm{t}), p(\overline{\mathrm{t}}), p\left(\ell^{+}\right), p\left(\ell^{-}\right)\right)$and $O_{3}=\varepsilon\left(p(\mathrm{~b}), p(\overline{\mathrm{b}}), p\left(\ell^{+}\right), p\left(\ell^{-}\right)\right)$, two

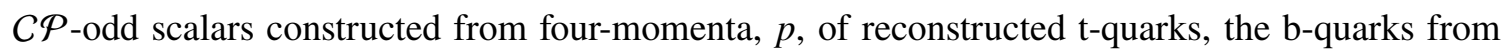
t-quark decays and the charged leptons from the W-boson decays, are used in CMS [29] to count the number, $N$, of tit-events at $\sqrt{s}=13 \mathrm{TeV}$ with positive and negative $O_{i}$, respectively, which lead to the two asymmetries $A_{i}=\frac{N\left(O_{i}>0\right)-N\left(O_{i}<0\right)}{N\left(O_{i}>0\right)+N\left(O_{i}<0\right)}, i \in\{1,3\}$. These asymmetries are sensitive to the presence of $C \mathcal{P}$-violating t-quark pair-production e.g. via a chromo-electric dipole moment (CEDM). The result of a likelihood fit after combining ee, $\mu \mu$ and $\mathrm{e}^{ \pm} \mu^{\mp}$ events is: $A_{1}=(2.4 \pm 2.8) \times 10^{-3}$, $A_{3}=(0.4 \pm 2.8) \times 10^{-3}$. Interpreted as CEDM, which is directly proportional to either $A_{i}$, gives the following results:

$\mathrm{CEDM}^{O_{1}}=0.58 \pm 0.69_{\text {stat }} \pm 0.70_{\text {syst }} 10^{-18} \mathrm{~g}_{\mathrm{s}} \mathrm{cm}, \mathrm{CEDM}^{O_{3}}=-0.01 \pm 0.72_{\text {stat }} \pm 0.58_{\text {syst }} 10^{-18} \mathrm{~g}_{\mathrm{s}} \mathrm{cm}$, where $g_{s}$ is the strong coupling constant, compatible with the vanishing SM-expectation.

\subsection{Fragmentation of b-quarks}

The b-quark fragmentation in Monte Carlo (MC) event generators like PYTHIA [14], HERWIG [15] or SHERPA [17] relies typically on tuning with $\mathrm{e}^{+} \mathrm{e}^{-} \rightarrow \mathrm{b} \overline{\mathrm{b}}$ data from LEP to the observed fraction, $x_{\mathrm{B}}=E_{\mathrm{B}} / E_{\text {beam }}$, of energy carried by a b-hadron, $E_{\mathrm{B}}$ over the beam energy, $E_{\text {beam }}$. For proton colliders, the energy of the fragmenting b-quark is not well defined. Recently, both ATLAS [30] and CMS [31] measured distributions based on the momentum fraction of b-hadrons (or their c-hadron daughters) from b-quark jets in t-quark decays, with the b-quark jet acting as proxy for the b-quark and hence sensitive to the b-quark fragmentation. CMS uses templates in the transverse momentum fraction carried by the b-hadron daughters $\left(\mathrm{J} / \Psi\right.$ or $\left.\mathrm{D}^{0}\right)$ with the Lund-Bowler fragmentation function shape coefficient, $r_{\mathrm{b}}$, which is used in PYTHIA, as template parameter. A simultaneous fit to $\mathrm{J} / \Psi$, tagged and un-tagged $\mathrm{D}^{0}$ distributions gives $r_{\mathrm{b}}^{\mathrm{CMS}}=0.858 \pm 0.037_{\text {stat }} \pm 0.031_{\text {syst }}$, in good agreement with the tunes from LEP. ATLAS compares transverse and longitudinal charged momentum fractions of b-quark-jets carried by the b-hadron to MC simulations with POWHEG interfaced to either PYTHIA or HERWIG and with events generated with SHERPA and finds good agreement with all three predictions.

\subsection{Polarisation of top-quarks}

QCD predicts unpolarised top-quarks in $\mathrm{pp} \rightarrow \mathrm{t} \overline{\mathrm{t}}$, but the $V-A$ structure of the Wtb-vertex leads to fully polarised top-quarks in the $t$-channel single-top production - along (against) the direction of the down-type spectator or incoming quark for top-quarks (antiquarks). ATLAS [32] defines a right-handed coordinate system in the t-quark rest-frame, with $\hat{z}^{\prime}$ along the spectator momentum direction, $\hat{y}^{\prime}$ orthogonal to $\hat{z}^{\prime}$ and the incoming light quark, and $\hat{x}^{\prime}$ orthogonal to $\hat{y}^{\prime}$ and $\hat{z}^{\prime}$. The charged lepton from the leptonic W-boson decay serves as analyser and all three polarisations (w.r.t. to the three defined coordinates) are measured. The SM expectations are $\simeq \pm 90 \%$ polarisation in the $\hat{z}^{\prime}$ direction, small values at $O( \pm 10 \%)$ in the $\hat{x}^{\prime}$ direction and 0 in the $\hat{y}^{\prime}$ direction, where non-zero values would indicate $C \mathcal{P}$-violation in the top-quark production. A 
template fit to the number of events grouped in bins of the sign of the cosines of the polar angles of the lepton momentum w.r.t. to the 3 axes $\left(2^{3}=8\right.$ bins) yields:

$P_{x^{\prime}}=0.01 \pm 0.18 ; P_{y^{\prime}}=-0.029 \pm 0.027 ; P_{z^{\prime}}=0.91 \pm 0.10$ for $\mathrm{t}$, and

$P_{x^{\prime}}=-0.02 \pm 0.20 ; P_{y^{\prime}}=-0.007 \pm 0.051 ; P_{z^{\prime}}=-0.79 \pm 0.16$ for $\overline{\mathrm{t}}$,

in good agreement with the SM expectations. Unfolded distributions in the cosines of the polar angles are used to constrain the complex Wilson-coefficient of the dimension-6 operator $O_{\mathrm{tW}}$ in effective field theory (EFT) extensions of the SM. At 95\% confidence level the real part, $C_{\mathrm{tW}} \in$ $[-0.7,1.5]$, and the imaginary part, $C_{i t \mathrm{~W}} \in[-0.7,0.2]$, are both found to be compatible with the SM expectation of 0 , respectively.

\subsection{Top-quark mass}

Direct and indirect methods to measure the mass of the top-quark are used at the LHC with increasing levels of precision [7] - both on the experimental and the theoretical side. The complementarity of the two approaches is ideal in the sense that systematic experimental and theoretical uncertainties impact the two methods differently. The most precise measurements to date are the Run-1 combinations of direct analyses in top-quark-pair events by ATLAS [33] and CMS [34] with $m_{\mathrm{t}}^{\text {ATLAS }}=172.69 \pm 0.48 \mathrm{GeV}$ and $m_{\mathrm{t}}^{\text {CMS }}=172.44 \pm 0.48 \mathrm{GeV}$, respectively. Recently, CMS [35] achieved to reach $0.8 \mathrm{GeV}$ precision in a direct measurement in $t$-channel single-top-quark lepton+jets events at $\sqrt{s}=13 \mathrm{TeV}$, which allows to probe a different colour-reconnection situation compared to $t \bar{t}$-events and allows to probe the mass difference of top-quarks and antiquarks. The lepton charge combined result is found to be $m_{\mathrm{t}}^{\text {CMS, single-top }}=172.13 \pm 0.32_{\text {stat }+ \text { prof }}{ }_{-0.7}^{+0.69}$ syst $\mathrm{GeV}$, with the first error for statistics and profiled systematics and the second for all other systematic uncertainties - dominated by signal and background modelling. The mass difference $\Delta\left(m_{\mathrm{t}}, m_{\overline{\mathrm{t}}}\right)=m_{\mathrm{t}}-m_{\overline{\mathrm{t}}}=0.83_{-1.01 \text { tot }}^{+0.77} \mathrm{GeV}$ is found to be compatible with the $\mathrm{SM}$ expectation of $0 \mathrm{GeV}$ within uncertainties. Indirect methods gained in precision in the measurement of the top-quark mass by expanding beyond the use of inclusive top-quark-pair production cross-sections to either $n$-differential cross-sections or shape variables as defined in [36]. A recent example for an $n$-differential cross-section based pole-mass is the di-lepton analysis by CMS [37] finding $m_{\mathrm{t}}^{\mathrm{CMS} \text {, pole }}=170.5 \pm 0.8 \mathrm{GeV}-$ the most precise indirect result to date. In $\bar{t}+1$ jet-events ATLAS [38] finds $m_{\mathrm{t}}^{\text {ATLAS, pole }}=171.1_{-1.0}^{+1.2} \mathrm{GeV}$, with the shape-variable defined in [36]. Both might suffer from additional theoretical uncertainties related to soft-gluon and Coulomb re-summation close to threshold [39] that are themselves not precisely known [40].

\section{Conclusions}

Top-quark production and properties in the decay of top-quarks are studied with high precision at the LHC. Experimental methods are getting more sophisticated, allowing for a rich spectrum of precision-measurements in the top-quark sector. Theoretical precision is often matched, sometimes surpassed and higher-order calculations are clearly helping in the modelling of top-quark production and decays. In general, the measurements are in good agreement with the SM expectations. So far, they do not hint at significant deviations from the SM. 


\section{References}

[1] ATLAS Collaboration, JINST 3, S08003 (2008).

[2] CMS Collaboration, JINST 3, S08004 (2008).

[3] LHCb Collaboration, JINST 3, S08005 (2008).

[4] ATLAS Collaboration, ATLAS-CONF-2021-013 (2021).

[5] ATLAS Collaboration, ATLAS-CONF-2021-003 (2021).

[6] CMS Collaboration, CMS-PAS-TOP-20-004 (2021).

[7] LHCTopWG, twiki.cern.ch/twiki/bin/view/LHCPhysics/LHCTopWGSummaryPlots (2021).

[8] M. Czakon, P. Fiedler and A. Mitov, Phys. Rev. Lett. 110, 252004 (2013).

[9] LHCb Collaboration, Phys. Lett. B 767, 110 (2017).

[10] LHCb Collaboration, JHEP 08, 174 (2018).

[11] ATLAS Collaboration, JHEP 01, 033 (2021).

[12] CMS Collaboration, arXiv:2108.02803, CMS-PAS-TOP-20-001 (2021).

[13] S. Alioli, P. Nason, C. Oleari and E. Re, JHEP 06, 043 (2010).

[14] T. Sjöstrand, S. Mrenna and P. Z. Skands, Comput. Phys. Commun. 178, 852 (2008).

[15] M. Bahr, et al., Eur. Phys. J. C 58, 639 (2008).

[16] J. Alwall, et al., JHEP 07, 079 (2014).

[17] Sherpa Collaboration, Sci. Post. Phys. 7, 034 (2019).

[18] S. Agostinelli, et al., Nucl. Instrum. Methods A 506, 250 (2003).

[19] J. Allison, et al., Nucl. Instrum. Methods A 835, 186 (2016).

[20] M. Grazzini, S. Kallweit and M. Wiesemann, Eur. Phys. J. C 78, 537 (2018).

[21] ATLAS Collaboration, Eur. Phys. J. C 81, 720 (2021).

[22] CMS Collaboration, arXiv:2109.01706, CMS-PAS-TOP-20-002 (2021).

[23] N. Kidonakis, Phys. Rev. D 82, 054018 (2010).

[24] N. Kidonakis, Summer School HQ Series HQ2013, 139 (2014).

[25] N. Kidonakis, PoS DIS2015, 170 (2015).

[26] ATLAS Collaboration, Nat. Phys. 17, 813 (2021). 
[27] LEP Electroweak Working Group, Phys. Rep. 532, 119 (2013).

[28] ATLAS and CMS Collaborations, JHEP 08, 051 (2020).

[29] CMS Collaboration, CMS-PAS-TOP-18-007 (2020).

[30] ATLAS Collaboration, ATLAS-CONF-2020-050 (2020).

[31] CMS Collaboration, CMS-PAS-TOP-18-012 (2021).

[32] ATLAS Collaboration, ATLAS-CONF-2021-027 (2021).

[33] ATLAS Collaboration, Eur. Phys. J. C 79, 290 (2019).

[34] CMS Collaboration, Phys. Rev. D 93, 072004 (2016).

[35] CMS Collaboration, CMS-PAS-TOP-19-009 (2021).

[36] S. Alioli, et al., Eur. Phys. J. C 73, 2438 (2013).

[37] CMS Collaboration, Eur. Phys. J. C 80, 658 (2020).

[38] ATLAS Collaboration, JHEP 11, 150 (2019).

[39] Y. Kiyo, et al., Eur. Phys. J. C 60, 375 (2009).

[40] J. Piclum and C. Schwinn, JHEP 03, 164 (2018). 